\title{
Qing-Kai-Ling Injection Acts Better Than Shen-Fu Injection in Enhancing the Antitumor Effect of Gefitinib in Resistant Non-Small Cell Lung Cancer Models
}

\author{
Ya-Ya Yu ${ }^{\mathbb{D}},{ }^{1,2}$ Yan-Juan Zhu $\mathbb{D}^{1,2,3,4}$ Ying Zou ${ }^{1}{ }^{1,5}$ Zhen-Zhen Xiao ${ }^{1 D},{ }^{1,2}$ Shuai Shi ${ }^{1,1,6}$ \\ Yi-Hong Liu $\mathbb{D}^{1,}$, ${ }^{1,2}$ Xue-Song Chang $\mathbb{D}^{1,2}$, Ya-Dong Chen $\mathbb{D}^{1,2}$ and Hai-Bo Zhang $\mathbb{D}^{1,2,3,4,7}$ \\ ${ }^{1}$ Department of Oncology, The Second Affiliated Hospital of Guangzhou University of Chinese Medicine, Guangzhou, China \\ ${ }^{2}$ Department of Oncology, Guangdong Provincial Hospital of Chinese Medicine, Guangzhou, China \\ ${ }^{3}$ Guangdong-Hong Kong-Macau Joint Lab on Chinese Medicine and Immune Disease Research, Guangzhou, China \\ ${ }^{4}$ Guangdong Provincial Key Laboratory of Clinical Research on Traditional Chinese Medicine Syndrome, Guangzhou, China \\ ${ }^{5}$ Department of Integrated Traditional and Western Medicine, \\ Affiliated Cancer Hospital and Institute of Guangzhou Medical University, Guangzhou, Guangdong, China \\ ${ }^{6}$ Department of Respiration Medicine, Luoyang First People's Hospital, Luoyang, Henan, China \\ ${ }^{7}$ State Key Laboratory of Dampness Syndrome of Chinese Medicine, Guangzhou, China
}

Correspondence should be addressed to Hai-Bo Zhang; haibozh@gzucm.edu.cn

Received 9 March 2021; Revised 13 August 2021; Accepted 18 September 2021; Published 4 October 2021

Academic Editor: Qing Ji

Copyright (C) $2021 \mathrm{Ya}$-Ya Yu et al. This is an open access article distributed under the Creative Commons Attribution License, which permits unrestricted use, distribution, and reproduction in any medium, provided the original work is properly cited.

Patients with EGFR gene mutation often obtain de novo resistance to epidermal growth factor receptor tyrosine kinase inhibitors (EGFR-TKIs) or develop secondary resistance to EGFR-TKIs after taking EGFR-TKI therapy. Traditional Chinese medicine (TCM) with different treatment principles, in combination with EGFR-TKIs, plays an important role in the treatment of cancers including resistant non-small cell lung cancer (NSCLC). However, inappropriate use of TCM herbs may induce resistance to gefitinib. Therefore, it is of a great value to evaluate which TCM treatment principle should be combined with EGFR-TKIs, and which one should be avoided, and find out the potential mechanisms. The lentiviral transfection assay was used for overexpression of PIK3CA mutation gene in PC-9 cells to construct PC-9-PIK3CA-mutation (PC-9-PIK3CA-M) cells. Cell proliferation, apoptosis, and the expression of EGFR/PI3K/AKT and EGFR/RAS/RAF/ERK in PC-9-PIK3CA-M and H1975 cells treated by the typical cooling-heat drug, Qing-kai-ling (QKL) and Tan-re-qing (TRQ), or the typical warming-yang drug, Shen-fu (SF) and gefitinib treatment, were detected by MTT, Annexin V/PI double labeling, and Western blot assays, respectively. Tumor xenograft and immunohistochemistry experiments were carried out to confirm the in vitro findings. PC-9-PIK3CA-M cells were less sensitive to gefitinib, when compared with PC-9 cells. QKL injection and TRQ injection, not SF injection, combined with gefitinib induced significantly increased cell growth inhibition and apoptosis in PC-9-PIK3CA-M and H1975 cells. SF injection antagonized the effect of gefitinib in promoting cancer cell apoptosis. QKL injection and TRQ injection increased the sensitivity of gefitinib by inhibiting the phosphorylation of AKT or ERK in H1975 and PC-9-PIK3CA-M cells. Similar findings were observed in vivo in H1975 xenograft mouse model. QKL and TRQ, with cooling-heat TCM treatment principle, should be combined with gefitinib in the treatment of NSCLC. Furthermore, warming-yang drug SF should be avoided to be used together with EGFR-TKIs.

\section{Introduction}

Epidermal growth factor receptor tyrosine kinase inhibitors (EGFR-TKIs) are the preferred treatment for intermediate to advanced stage non-small cell lung cancer (NSCLC) in patients with EGFR gene mutation [1,2]. However, 20 30\% of these patients obtained de novo resistance to the first-line or second-line EGFR-TKIs [3, 4]. Moreover, even for the exclusively EGFR-mutant advanced NSCLC patients, the median progression-free survival (PFS) was only 9 13 
months for those who received the first-line EGFR-TKI therapy and developed secondary resistance $[1,2]$. Therefore, effective therapies to increase the sensitivity to gefitinib for patients in these situations are urgently required.

Traditional Chinese medicine (TCM) therapies have been widely used in cancer treatment including NSCLC in China. Although clinical trials have reported the efficacy of some TCM decoction or patent prescription, with different TCM treatment principles, in combination with EGFRTKIs, most of them were with small sample size and low quality [5]. What is more, a case report by Hwang et al. indicated that some TCM herbs may even induce resistance to gefitinib [6]. This phenomenon is similar to that in the western medicine that certain drugs, such as antifungal drugs, could induce primary and acquired resistance of EGFR-TKI in lung cancer [7]. Therefore, it is of a great value to explore which TCM treatment principle should be combined with EGFR-TKIs and which principle should be avoided to be combined with EGFR-TKIs using the TCM theory. We have previously demonstrated that EGFR gene mutated NSCLC patients, who are sensitive to EGFR-TKIs, were more likely with Yin-cold (YC) TCM syndrome type [8]. Besides, the most common side effects of EGFR-TKIs are red acneiform rashes [1-4, 9], with thirsty, red, and dry tongue and yellow tongue coating, that are typical symptoms and signs of Yang-heat (YH) syndrome type. Therefore, EGFR-TKIs may affect the TCM warming-yang symptoms. We also noticed that patients with primary or secondary resistance to EGFR-TKIs have more chance to be diagnosed with $\mathrm{YH}$ syndrome type. According to TCM theory, we propose a hypothesis that cooling-heat, rather than warmingyang TCM treatment principle, should be used when combined with EGFR-TKIs and may help to improve the efficacy of EGFR-TKIs in resistant NSCLC.

In order to confirm this hypothesis, we used Qing-kailing (QKL) injection/oral solution and Shen-fu (SF) injection/SF decoction, representing typic cooling-heat and warming-yang drugs, respectively, with no known anticancer effect. QKL is a notable antipyretic preparation and is widely used for the treatment of the upper respiratory inflammation, viral encephalitis, pneumonia, and high fever in clinical practice [10] and is listed in the Chinese Pharmacopoeia (State Pharmacopoeia Committee, 2020). SF has been used for nearly 30 years in China for patients with YC syndrome type. It can attenuate postresuscitation brain edema, myocardial dysfunction, and lung injury [11]. Gefitinib was combined with QKL or SF solution, both in vitro and in vivo, to detect the interactions and underlying mechanisms. Another classical TCM formulation, Tan-reqing (TRQ) injection, also termed as cooling-heat traditional Chinese medicine, is commonly used to treat acute upper respiratory tract infection and early stage pneumonia in clinical practice [12]. In this study, we used TRQ injection to investigate whether other cooling-heat traditional Chinese medicines had synergic effects with gefitinib. PC-9-PIK3CAmutation (PC-9-PIK3CA-M, PC-9 with stable overexpression of PIK3CA mutated gene, 19Del/PIK3CA) and H1975 (EGFR 19Del/T790M) cell lines were selected for the study, because PIK3CA [13] and T790M [14] were the most important genetic covariations in primary and secondary EGFR-TKIs-resistant patients, respectively.

\section{Materials and Methods}

2.1. Preparation of Drugs. Gefitinib was bought from Selleck Co., Ltd (Shanghai, China). QKL injection used in vitro was bought from Yi-Sheng Pharmaceutical Co., Ltd (Ji'an, China) and QKL oral solution applied in vivo was purchased from Ming-Xing Pharmaceutical Co., Ltd (Guangzhou, China), SF injection used in vitro was purchased from Yaan San-Jiu Pharmaceutical Co., Ltd (Sichuan, China), and TRQ injection applied in vitro was bought from Kai-Bao Pharmaceutical Co., Ltd (Shanghai, China). Components of QKL include Isatis tinctoria L., Gardenia jasminoides J.Ellis, Lonicera japonica Thunb., Concha Margaritifera Usta, powdered buffalo horn, baicalin, hyodeoxycholic acid, and cholic acid. The constituents of QKL prescription are shown at Table 1. Different batches of QKL injection were analyzed in consistency studies (chromatograms) using high performance liquid chromatography (HPLC) [15]. SF is prepared by the well-known traditional Chinese herbs Panax Ginseng and Radix Aconiti Carmichaeli. The consistency of the quality of SF injection over different batches has been ensured by fingerprint technology, thereby identifying the authenticity of drugs [11]. TRQ injection is produced from the raw material of five herbs: Scutellariae Radix, Fel selenarcti, Cornu naemorhedi, Lonicerae japonicae flos, and forsythia fructus. The consistency of the quality of TRQ injection over different batches has been ensured by a reliable ultra-high performance liquid chromatography-mass spectrometry technology (UHPLC-MS) [16]. The components of herbs of SF decoction applied in the in vivo experiments, prepared Radix Panax Ginseng and Radix Aconiti Carmichaeli, were purchased from Kang-Mei Pharmaceutical Co., Ltd (Guangzhou, China). Seventy-five grams (g) of Panax Ginseng and $150 \mathrm{~g}$ Radix Aconiti Carmichaeli soaked in water were boiled twice; then the mixed solution was concentrated into $250 \mathrm{~mL}$ in the rotary evaporator (IKA ${ }^{\circledR} \mathrm{RV}$ 10 Basic), with a concentration of $0.9 \mathrm{~g} / \mathrm{mL}$ crude drug. The constituents of SF prescription are shown at Table 2.

2.2. Reagents. 4,5-Dimethylthiazol-2, 5-diphenyl-2-H-tetrazolium bromide (MTT) was bought from MP Biomedicals (California, USA). Annexin V/propidium iodide (PI) apoptosis kit was purchased from MultiSciences Biotech Co., Ltd (Hangzhou, China). BCA protein assay kit was bought from Thermo Fisher Scientific, Inc (ML, USA). Rabbit anti-human PIK3CA, EGFR, phospho-EGFR (p-EGFR), AKT, p-AKT, ERK, and p-ERK monoclonal antibodies (mAb) and horseradish peroxidase (HRP) conjugated anti-rabbit antibody were purchased from Cell Signaling Technology, Inc (MA, USA). Electro-Chemi-Luminescence (ECL) reagent was purchased from Millipore Corporation (MA, USA).

2.3. Cell Culture. Human PC-9 cells (EGFR 19Del) and H1975 cells (EGFR 19Del/T790M) were obtained from the Cell Line Bank at the Laboratory Animal Center of Sun 
TABle 1: Constituents of Qing-kai-ling prescription.

\begin{tabular}{lc}
\hline Common name & Weight $(\mathrm{g})$ \\
\hline Isatidis radix & 200.00 \\
Lonicerae japonicae flos & 60.00 \\
Gardeniae fructus & 25.00 \\
Buffalo horn & 25.00 \\
Concha margaritifera & 50.00 \\
Baicalin & 5.00 \\
Cholic acid & 3.25 \\
Hyodeoxycholic acid & 3.75 \\
\hline
\end{tabular}

TABle 2: Constituents of Shen-Fu prescription.

\begin{tabular}{lc}
\hline Common name & Weight $(\mathrm{g})$ \\
\hline Radix ginseng & 75.00 \\
Radix aconiti lateralis preparata & 150.00 \\
\hline
\end{tabular}

Yat-sen University (Guangzhou, China) and the Macao University of Science and Technology (Macao, China), respectively. Cells were maintained in RPMI-1640 medium (Gibco, MA, USA) supplemented with 10\% FBS (Gibco, MA, USA) and $1 \%$ penicillin-streptomycin sulfate (Gibco, MA, USA) and incubated at $37^{\circ} \mathrm{C}$ with $5 \% \mathrm{CO}_{2}$.

2.4. The Construction of PC-9-PIK3CA-M Cell Line Using Lentivirus Transfection. The PC-9 cells were planted into a 12 -well plate with the cell density of $1 \times 10^{5} /$ well. The cells were transfected with $10 \mathrm{MOI}$ of lentivirus containing PIK3CA mutation gene (E545K) overexpressing plasmid after $24 \mathrm{~h}$ of incubation. When the cells reached $80 \%-90 \%$ confluence, PC- 9 cells were passaged and $2 \mu \mathrm{g} / \mathrm{mL}$ puromycin was added to the media for maintenance culture to select the virus infected cells. Then Western blotting analysis was used to confirm the overexpression of PIK3CA and its downstream protein expression in PC-9-PIK3CA-M cells.

2.5. Cell Viability Assay. MTT assay was used to measure cell viability. Cells were planted in 96-well culture plates at the density of $5 \times 10^{3} /$ well. After $24 \mathrm{~h}$ of incubation, cells were treated with drugs alone or in combination for $48 \mathrm{~h}$ and $72 \mathrm{~h}$. Then the cells were incubated with $0.5 \mathrm{mg} / \mathrm{mL}$ MTT at $37^{\circ} \mathrm{C}$ for $4 \mathrm{~h}$. The dimethyl sulfoxide (DMSO) was added and the light absorbance at $570 \mathrm{~nm}$ of each well was measured spectrophotometrically using a microplate reader (VICTOR $\mathrm{X} 5$, PerkinElmer, USA). The cell proliferation inhibition rate was determined as follows: inhibition rate $=\left(\mathrm{OD}_{570 \mathrm{~nm}}\right.$ value of the control group - $\mathrm{OD}_{570 \mathrm{~nm}}$ value of the experimental group)/OD $\mathrm{OD}_{570 \mathrm{~nm}}$ value of the control group.

2.6. Apoptosis Assay. Cells were planted in 6-well culture plates at a density of $3 \times 10^{5}$ cells/well. After $24 \mathrm{~h}$ of incubation, cells were treated with drugs alone or in combination, with the optimal concentration and exposure time according to the MTT assay results. After removing the medium, cells were trypsinized with EDTA-free trypsin solution, harvested, and then resuspended in $500 \mu \mathrm{L}$ Binding Buffer $(1 \times)$ with $5 \mu \mathrm{L}$ Annexin V-FITC and $10 \mu \mathrm{L}$ PI. After incubation for $5 \mathrm{~min}$ at room temperature in the dark, the samples were analyzed using a flow cytometer (Beckman Coulter FC500, USA).

2.7. Western Blot Analysis. The cells were plated in 6-well culture plates at a density of $3 \times 10^{5}$ cells/well. After $24 \mathrm{~h}$ of incubation, cells were treated with drugs alone or in combination. After $48 \mathrm{~h}$ treatment, the cells were lysed in lysis buffer. The concentration of proteins was determined, and 20-30 $\mu \mathrm{g}$ protein of each group was resolved on an $10 \%$ denatured SDS-polyacrylamide gel and transferred onto a PVDF membrane (Millipore, MA, USA). After blocking nonspecific binding sites with $5 \%$ milk, the membranes were incubated with rabbit anti-human EGFR, p-EGFR, AKT, p-AKT, ERK, and p-ERK monoclonal antibodies overnight at $4^{\circ} \mathrm{C}$. Then the membranes were incubated with HRP conjugated anti-rabbit antibody for $1 \mathrm{~h}$ at room temperature. Finally, signals were detected using a freshly prepared ECL solution and the ChemiDoc XRS + System (Bio-Rad, Hercules, CA, USA). ImageLab software (version 4.0) was used to calculate the expression of each protein, which was normalized by GAPDH.

2.8. Determination of the Antitumor Effect in Nude Mice. The animal experiment was approved by the Animals Research Committee of Guangdong Provincial Hospital of Chinese Medicine (NO.2017050). Sixty Female BALB/c nude mice $(18-20 \mathrm{~g})$ were obtained from the Laboratory Animal Center of Southern Medical University (Guangzhou, China, License NO. 44002100006205) and kept in the Animal Center of Guangdong Provincial Hospital of Chinese Medicine (License NO. SYXK (yue) 2013-0094). H1975 cells $\left(6 \times 10^{6} /\right.$ mouse $)$ were subcutaneously inoculated into the right forelimb of the nude mice. Tumor growth was measured with the longest diameter (a) and the shortest diameter vertical to a (b). Tumor volume was calculated using the formula, $\mathrm{V}=\pi \mathrm{ab}^{2} / 6$. When the tumors reached the size over $150 \mathrm{~mm}^{3}$, the mice were randomly divided into 6 groups $(n=10)$ : control (saline solution $0.2 \mathrm{~mL}$ ), gefitinib ( $1 \mathrm{mg}$, in $0.2 \mathrm{~mL}$ saline solution), QKL oral solution $(0.25 \mathrm{~mL})$, SF decoction $(0.2 \mathrm{~mL})$, gefiti$\mathrm{nib}+\mathrm{SF}$, and gefitinib $+\mathrm{QKL}$ group. The compounds were administered by gavage once a day for consecutive 21 days. The daily dosage of each drug for nude mice (with an average weight of $20 \mathrm{~g}$ ) was obtained based on the daily dosage for human in clinical and the human-mouse transfer formula: Animal dose $=$ Human dose $\times(\mathrm{HKm} /$ $\mathrm{AKm}$ ), where $\mathrm{HKm}$ and $\mathrm{AKm}$ represent the $\mathrm{Km}$ factor of human (37) and mouse (3) [17]. The daily dosages for human (with average weight of $60 \mathrm{~kg}$ ) are $250 \mathrm{mg}$ of gefitinib, $60 \mathrm{ml}$ of QKL oral solution, and $45 \mathrm{~g}$ crude drug of SF decoction. The mice were sacrificed 21 days after the treatment using isoflurane and the tumors were excised and weighed and were later placed in paraformaldehyde (4\%) for immunohistochemistry (IHC). 
2.9. IHC. For IHC staining, the sections were applied to block endogenous peroxide activity and then boiled in $0.01 \mathrm{M}$ citrate buffer ( $\mathrm{pH}$ 6.0) with an autoclave. Tissue sections were incubated with rabbit anti-human EGFR (1: 100), p-EGFR (1:100), AKT (1:200), p-AKT (1:50), ERK $(1: 200)$, or $\mathrm{p}$-ERK $(1: 100)$ monoclonal antibodies at $4^{\circ} \mathrm{C}$ overnight. The sections were incubated with HRP conjugated anti-rabbit antibody after washing, and the peroxidase reaction was developed with diaminobenzidine substrate kit (Zhongshan Golden Bridge-Bio, Beijing, China). Hematoxylin (Dingguo Changsheng Biotechnology Co., Ltd, Beijing, China) was used for nuclear staining. Image-ProPlus software (version 5) was used to calculate the ratio of integrated optical density (IOD) to area (IOD/Area).

2.10. Statistics. Statistical analysis was performed using SPSS 19.0 statistical software (SPSS, Inc., Chicago, USA). The in vitro experiments were performed three times, independently. All data were presented as the mean \pm standard deviation (SD). Differences between groups were assessed by two-tailed $t$-test, one-way analysis of variance, or analysis of variance for repeated measuring data, and least significant difference (LSD) $t$ test was used for multiple comparisons. $P<0.05$ was considered to indicate a statistically significant difference. $q$ value method was used to evaluate the combination effect of gefitinib and QKL/SF, and it was calculated using the equation: $q=\mathrm{EAB} /(\mathrm{EA}+\mathrm{EB}-\mathrm{EA} \times \mathrm{EB})$, where $\mathrm{EA}, \mathrm{EB}$, and $\mathrm{EAB}$ were the inhibition effect of gefitinib, QKL/SF, and gefitinib combined with QKL/SF, respectively [18]. A q value of 1.15 or more is considered as synergism, $q<0.85$ as antagonism, and the value between 0.85 and 1.1.5 as additive effect.

\section{Results}

3.1. PC-9-PIK3CA-M Cells Activated PIK3CA-AKT Signal Pathway and Became Less Sensitive to Gefitinib due to Upregulated Phosphorylation of AKT Protein. Because there was no NSCLC cell line expressing both EGFR 19Del gene and PIK3CA mutation gene, we used the lentiviral transfection to stably overexpress PIK3CA mutation gene in PC9 cell line to obtain the PC-9-PIK3CA-M cell model. PIK3CA gene mutation activates the PIK3CA/AKT/mTOR signal pathway [19]. The PIK3CA and p-AKT were overexpressed in PC-9-PIK3CA-M cells (Figure 1(a), $P<0.05$ ). MTT results indicated that PC-9-PIK3CA-M cells were less sensitive to gefitinib, when compared with PC-9 cells (Figure 1(b); $P<0.05)$. With the same dose treatment of gefitinib, the expression of p-AKT was significantly higher in PC-9-PIK3CA-M cells than that in PC-9 cells (Figure 1(c); $P<0.05)$. These results indicated that PC-9-PIK3CA-M were less sensitive to gefitinib due to upregulated phosphorylation of AKT protein.

3.2. QKL Injection and TRQ Injection, but Not SF Injection, Significantly Inhibited the Cancer Cell Viability In Vitro. We first screened the concentration of gefitinib. According to the MTT assay, $15 \mu \mathrm{M}$ of gefitinib for PC-9-PIK3CA-M cells and $25 \mu \mathrm{M}$ for $\mathrm{H} 1975$ cells were used in the experiments with 48 hours of drug exposure, and $15 \mu \mathrm{M}$ gefitinib for PC9-PIK3CA-M cells and $20 \mu \mathrm{M}$ for $\mathrm{H} 1975$ cells were used to expose the cells to the drugs for 72 hours.

As shown in Figure 2(a), in PC-9-PIK3CA-M and H1975 cells, the concentration of $0.6 \%, 0.7 \%, 0.8 \%$, and $0.9 \% \mathrm{QKL}$ injection combined with gefitinib induced significantly increased antiproliferation after the treatment for both $48 \mathrm{~h}$ and $72 \mathrm{~h}$, when compared with the gefitinib group and QKL group (Figures 2(a) and 2(b); $P<0.05$ ). However, there was no significant difference in viability between gefitinib group and SF + gefitinib group in PC-9-PIK3CA-M cells after the treatment with SF at any concentration for both $48 \mathrm{~h}$ and $72 \mathrm{~h}$ (Figure $2(\mathrm{a}) ; P<0.05)$. The same phenomenon was observed in $\mathrm{H} 1975$ cells (Figure 2(b); $P<0.05$ ). Moreover, it was confirmed that another cooling-heat traditional Chinese medicine, TRQ injection, also had synergy effect with gefitinib (Figure 2(c); $P<0.05$ ).

3.3. QKL Injection Synergistically Increased the Proapoptotic Effect of Gefitinib, While SF Injection Antagonized the Proapoptotic Effect of Gefitinib In Vitro. QKL + gefitinib group significantly increased the rate of apoptosis, when compared with the control, gefitinib, and QKL groups in both the PC-9PIK3CA-M and H1975 cells (Figure 3; P<0.05). However, the differences in apoptotic rates were not significant between the $\mathrm{SF}+$ gefitinib group and gefitinib group (Figure 3; $P<0.05)$ in PC-9-PIK3CA-M cells as well as in H1975 cells. Synergism effect of QKL injection and gefitinib on apoptosis was seen in PIK3CA-M cells $(q=2.08)$, and QKL injection and gefitinib have additive effect in H1975 cells $(q=0.99)$ using the $q$ value method. On the contrary, SF injection antagonized the proapoptotic effect of gefitinib in both PIK3CA-M $(q=0.71)$ and H1975 cells $(q=0.64)$.

\subsection{QKL/TRQ Injection, but Not SF Injection, in Combination} with Gefitinib Inhibits the AKT or ERK Protein Expression In Vitro. The protein expression levels of $\mathrm{p}$-AKT were significantly lower in gefitinib + QKL group in PC-9-PIK3CA$M$ cells, comparing with the control group and the gefitinib group (Figure $4 ; P<0.05$ ). No significant differences were seen in $\mathrm{p}$-AKT expression among the control group, gefitinib, and gefitinib $+S F$ groups. Besides, the protein expression of $\mathrm{p}$-ERK was significantly lower in gefitinib $+\mathrm{QKL}$ group in PC-9-PIK3CA-M cells, comparing with the control group and the gefitinib group (Figure $4 ; P<0.05$ ). Besides, no significant differences were seen in the p-ERK expression between the gefitinib and gefitinib + SF groups. These results suggested that the downregulated phosphorylation of AKT and ERK protein may contribute to the synergic effect of QKL injection and gefitinib in PC-9-PIK3CA-M cells. In H1975 cell line, the p-AKT protein expression was much lower in QKL + gefitinib group, when compared with both the control group and the gefitinib group (Figure 4; $P<0.05)$. There was no significant difference seen in the p-AKT expression between SF + gefitinib group and gefitinib group. However, the expression of EGFR, p-EGFR, AKT, ERK, and p-ERK has no significant difference in the gefitinib, QKL+gefitinib, and SF+gefitinib groups. These 


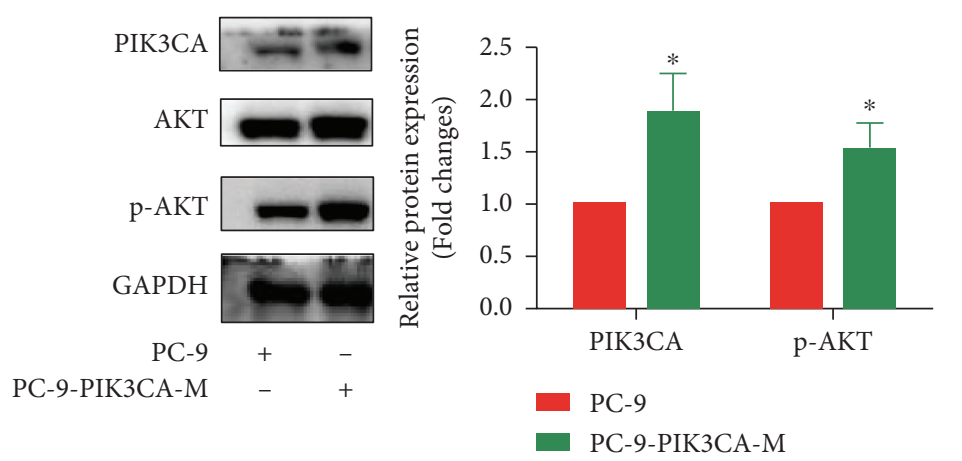

(a)

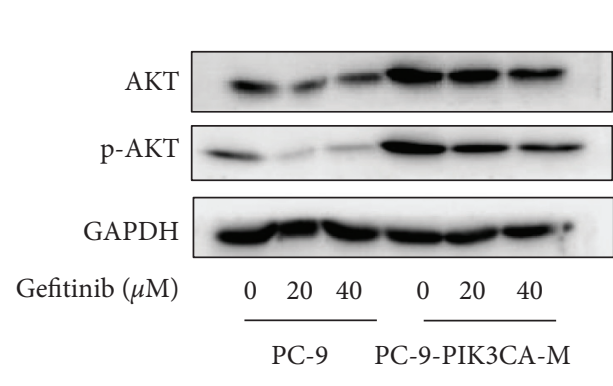

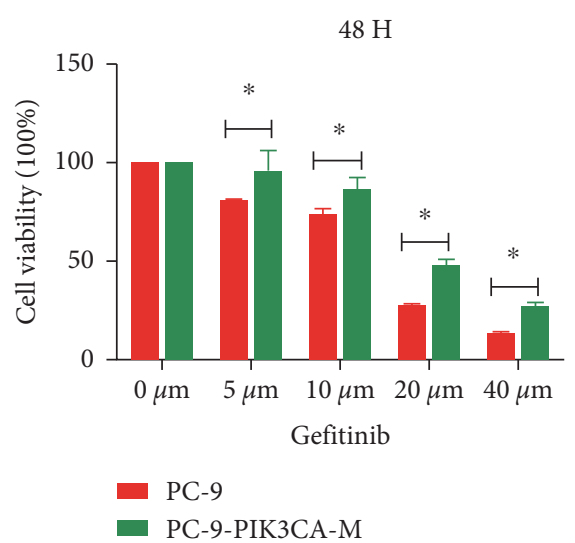

(b)

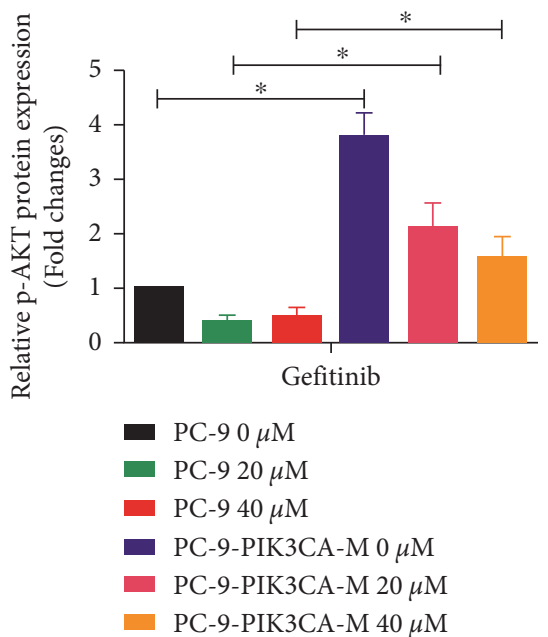

FIgURE 1: PC-9-PIK3CA-M cells are less sensitive to gefitinib due to the activated PIK3CA-AKT signal pathway: (a) Western blot assay detecting the protein expression of PIK3CA, AKT, and p-AKT in PC-9 and PC-9-PIK3CA-M cells. (b) MTT assay detecting the cell viability after the treatment with gefitinib in PC-9 and PC-9-PIK3CA-M cells. (c) Western blot assay detecting the protein expression of AKT, p-AKT after the treatment with gefitinib in PC-9 and PC-9-PIK3CA-M cells. Data are presented as the mean \pm standard deviation of three independent experiments. ${ }^{*} P<0.05$ vs. control group. PC-9-PIK3CA-M, PC-9-PIK3CA-mutation.

results suggested that the downregulation of p-AKT may contribute to the synergistic effect between QKL injection and gefitinib in H1975 cells. These results suggested that the downregulated phosphorylation of AKT protein may contribute to the synergic effect of QKL injection and gefitinib in vitro.

Furthermore, to examine whether other cooling-heat traditional Chinese medicines, other than QKL, have inhibitory effects on EGFR/AKT and EGFR/ERK signaling pathways, we detected the effects of TRQ injection and gefitinib on the expression of EGFR, AKT, and ERK proteins. The results indicated that the downregulated phosphorylation of ERK protein contributed to the synergism effect between QKL injection and gefitinib in PC-9-PIK3CAcells, while the downregulated phosphorylation of AKT protein might contribute to the synergic effect of QKL injection and gefitinib in H1975 cells.
3.5. QKL Oral Solution, Not SF Decoction, Enhanced the Antitumor Effect of Gefitinib via Regulating p-AKT In Vivo. QKL, gefitinib, and QKL + gefitinib inhibited tumor growth with significantly smaller tumor volume and lighter tumor weight, compared with those in control group (Figures 5(a) and 5(b); $P<0.05)$. There was a significant difference in tumor volume and tumor weight between $\mathrm{QKL}+$ gefitinib group and gefitinib group (Figures 5(a) and 5(b); $P<0.05$ ). However, there was no significant difference between $\mathrm{SF}+$ gefitinib group and gefitinib group, or $\mathrm{SF}+$ gefitinib group and control group.

In H1975 xenograft transplanted nude mice, the p-AKT protein expression was much lower in the $\mathrm{QKL}+$ gefitinib group compared with that in the control group (Figures 5(c) and 5(d); $P<0.05)$ as well as the gefitinib group (Figures 5(c) and $5(\mathrm{~d}) ; P<0.05)$. There was no significant difference in the $\mathrm{p}$-AKT protein expression between $\mathrm{SF}+$ gefitinib group and 
PC-9-PIK3CA-M QKL+Gefitinib 48 H

PC-9-PIK3CA-M SF+Gefitinib $48 \mathrm{H}$
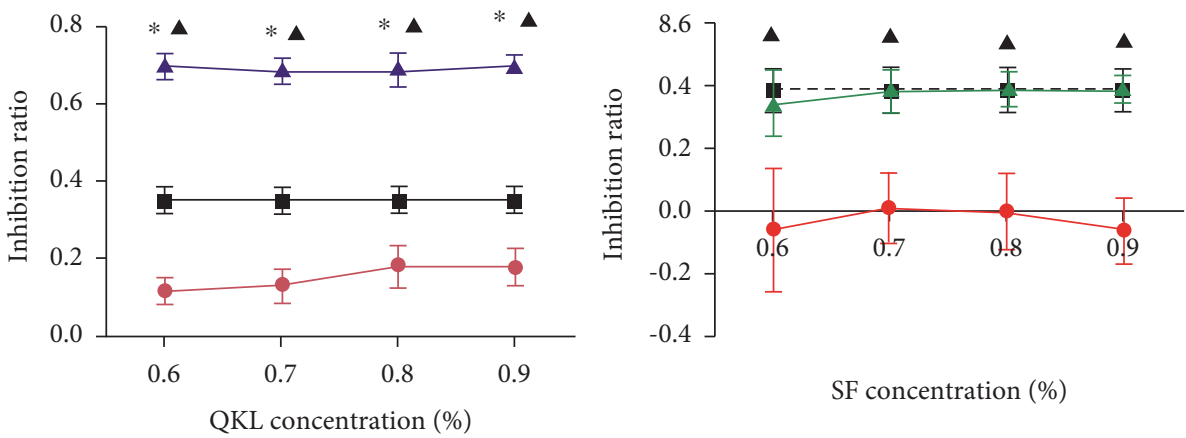

SF concentration (\%)

$\rightarrow-$ QKL

$\rightarrow$ Gefitinib $(15 \mu \mathrm{M})$

$\neg$ QKL+Gefitinib $(15 \mu \mathrm{M})$

PC-9-PIK3CA-M QKL+Gefitinib 72 H

- SF

- - Gefitinib $(15 \mu \mathrm{M})$

$\neg$ SF+Gefitinib $(15 \mu \mathrm{M})$
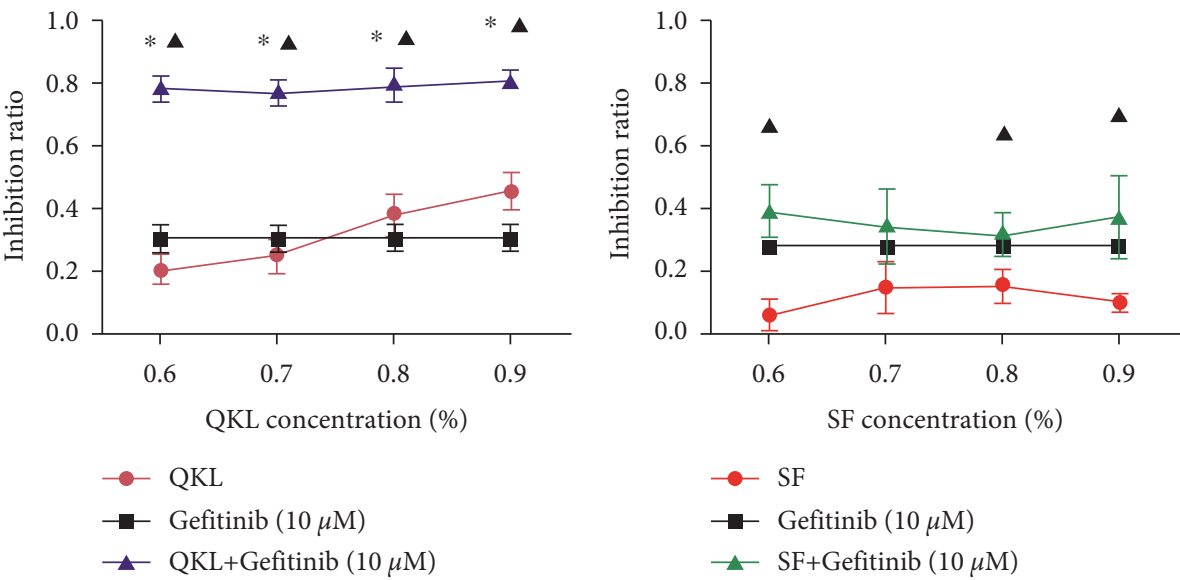

(a)

Figure 2: Continued. 
H1975 QKL+Gefitinib 48 H

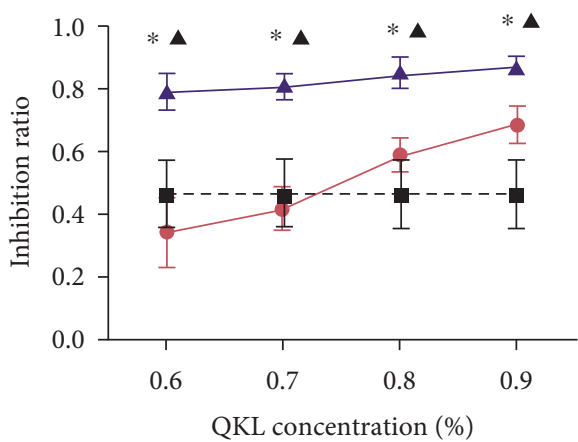

$\longrightarrow$ QKL

-匹- Gefitinib $(25 \mu \mathrm{M})$

$\simeq$ QKL+Gefitinib $(25 \mu \mathrm{M})$

H1975 QKL+Gefitinib 72 H

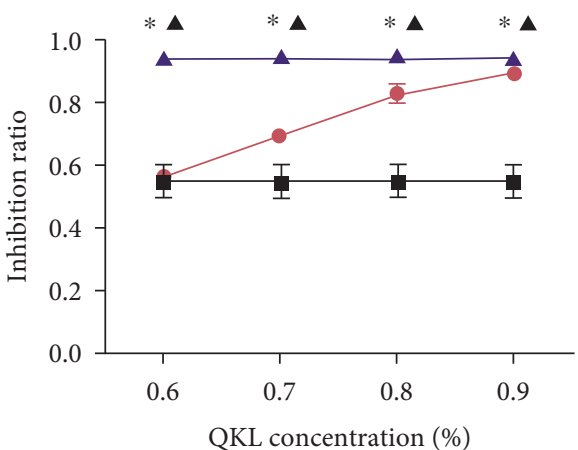

$\longrightarrow$ QKL

$\rightarrow$ Gefitinib $(20 \mu \mathrm{M})$

$\neg$ QKL+Gefitinib $(20 \mu \mathrm{M})$

PC-9-PIK3CA-M TRQ+Gefitinib $48 \mathrm{H}$

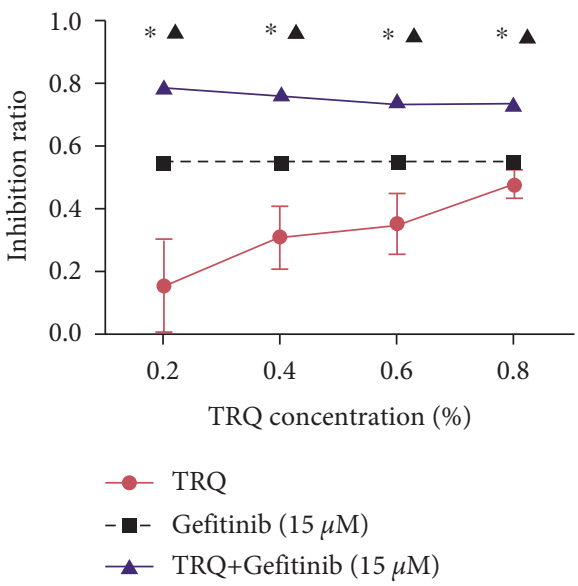

H1975 SF+Gefitinib 48 H

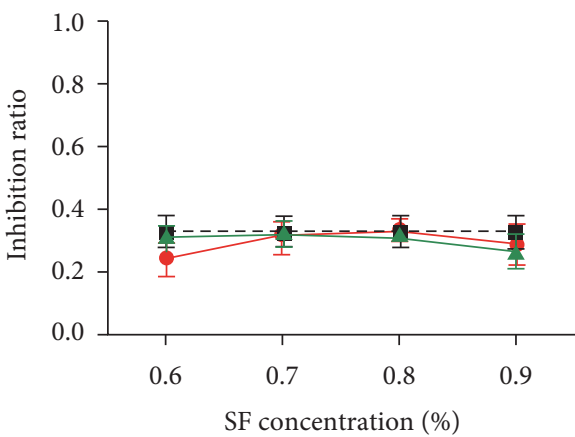

$\longrightarrow \mathrm{SF}$

-ם- Gefitinib $(25 \mu \mathrm{M})$

$\simeq$ SF+Gefitinib $(25 \mu \mathrm{M})$

H1975 SF+Gefitinib 72 H

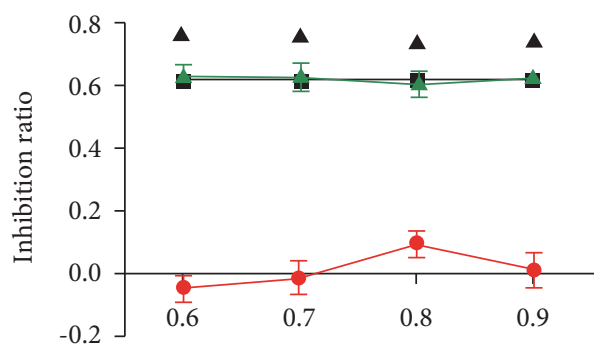

SF concentration (\%)

$\rightarrow$ SF

$\rightarrow$ Gefitinib $(20 \mu \mathrm{M})$

$\neg$ SF+Gefitinib $(20 \mu \mathrm{M})$

(b)

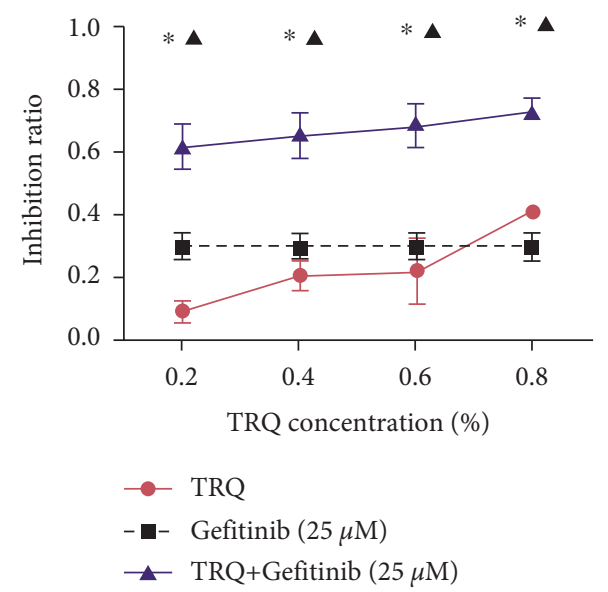

(c)

FIGURE 2: QKL injection and TRQ injection, but not SF injection, increased the sensitivity of gefitinib in PC-9-PIK3CA-M and H1975 cells: (a) MTT assay on the cell viability after the treatment with QKL/SF and gefitinib for $48 \mathrm{~h}$ and $72 \mathrm{~h}$ in PC-9-PIK3CA-M cells. (b) MTT assay detecting the cell viability after the treatment with QKL/SF and gefitinib for $48 \mathrm{~h}$ and $72 \mathrm{~h}$ in $\mathrm{H} 1975 \mathrm{cells}$. (c) MTT assay detecting the cell viability after the treatment with TRQ and gefitinib for $48 \mathrm{~h}$ in PC-9-PIK3CA-M and H1975 cells. Data are presented as the mean \pm standard deviation of three independent experiments. ${ }^{*} P<0.05$ vs. gefitinib alone, ${ }^{\mathbf{A}} P<0.05$ vs. QKL/SF alone. QKL, Qing-kai-ling injection; SF, Shen-fu injection; TRQ, Tan-re-Qing injection. 

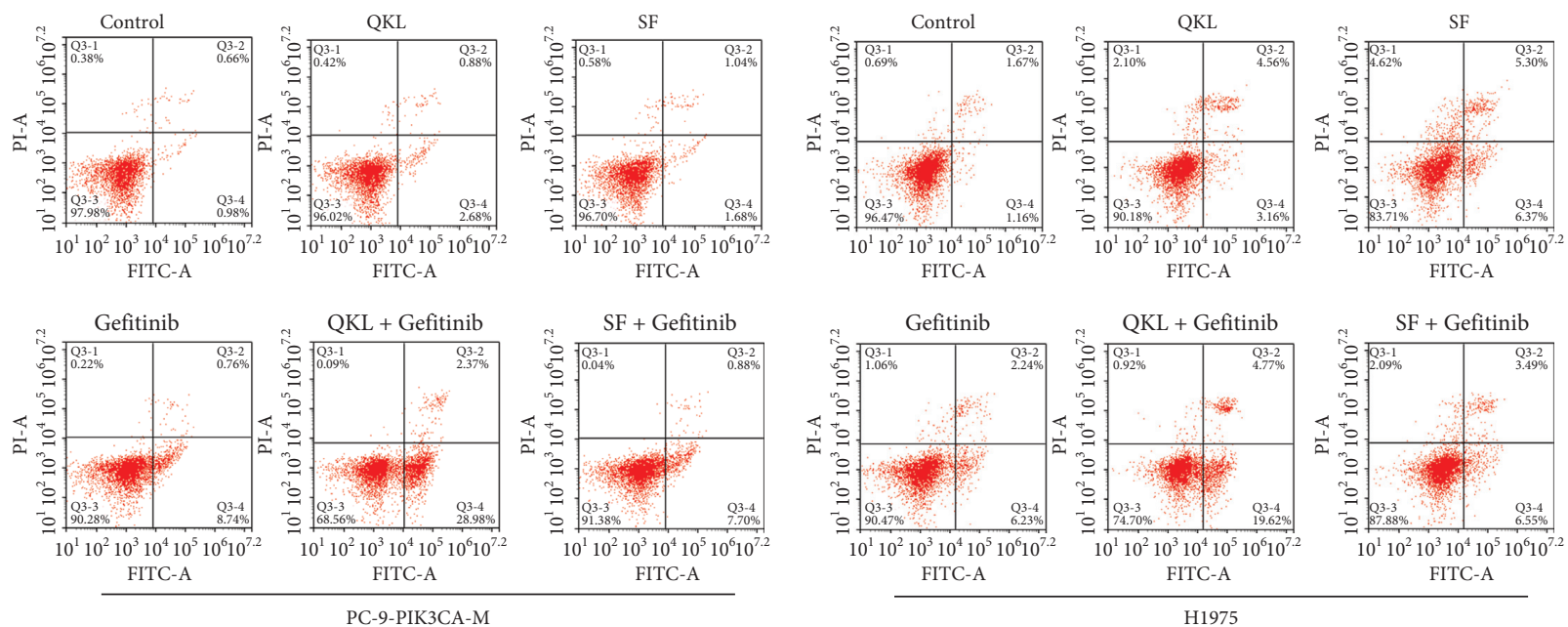

(a)

(b)

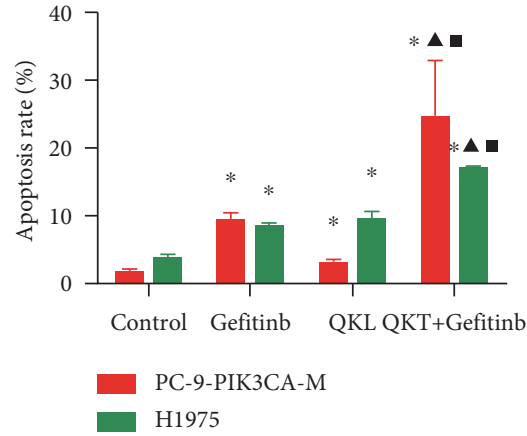

(c)

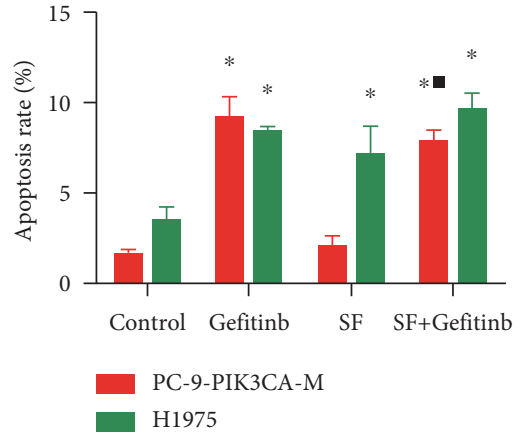

(d)

FIGURE 3: QKL injection increased, and SF injection antagonized the proapoptotic effect of gefitinib in PC-9-PIK3CA-M and H1975 cells: (a) apoptosis assay to assess the combination effect of gefitinib and QKL/SF for $48 \mathrm{~h}$ in PC-9-PIK3CA-M cells. (b) Apoptosis assay to assess the combination effect of gefitinib and QKL/SF for $48 \mathrm{~h}$ in $\mathrm{H} 1975$ cells. (c) The statistics of apoptotic rate induced by gefitinib and QKL in PC-9-PIK3CA-M and H1975 cells. (d) The statistics of apoptotic rate induced by gefitinib and SF in PC-9-PIK3CA-M and H1975 cells. Data are presented as the mean \pm standard deviation of three independent experiments. ${ }^{*} P<0.05$ vs. control group, ${ }^{\wedge} P<0.05$ vs. gefitinib group, and $P<0.05$ vs. QKL/SF group. QKL, Qing-kai-ling injection; SF, Shen-fu injection.

gefitinib group, or SF + gefitinib group and control group. These results demonstrated that the downregulated phosphorylation of AKT may contribute to the additive effect of QKL and gefitinib in vivo [18].

\section{Discussion}

TCM has been widely used in at least 78 countries [20], especially in East Asia. Many NSCLC patients receive TCM treatments when taking EGFR-TKIs, with the wish to delay or reverse EGFR-TKI resistance. A meta-analysis on a biomedical literature database showed that using TCM in combination with EGFR-TKIs was significantly superior to the use of EGFR-TKI alone in total response rate, quality of life improvement, and one-year survival rate of patients with NSCLC $(P<0.05)$ [21]. TCM combination therapy may increase therapeutic effects and reduce toxicity when combined with EGFR-TKIs for advanced NSCLC as well, indicating that this combination maximizes the duration of the EGFR- TKI treatment for NSCLC patients. However, inappropriate use of TCM may antagonize the effect of
EGFR-TKIs [6]. In a case report, the NSCLC patient was treated with gefitinib as the first-line treatment and had progressively increasing shortness of breath and imaging examination revealed progression of her disease within 9 weeks of gefitinib treatment [6]. Later on, it was found that multiple complementary herbal medicines such as ginseng, taken with gefitinib by the patients without the permission of the doctor, led to the disease progression, and her disease was finally relieved after taking gefitinib alone for 30 weeks [6]. In the real world of TCM clinical practice, clinicians first differentiate TCM syndrome types, then decide the TCM treatment principle, and finally write out a TCM prescription. Therefore, deciding TCM treatment principle is an important step, acting as bridge between TCM syndrome diagnosis and final TCM prescription. For this reason, we have focused on finding out the appropriate TCM treatment principles in the combinational use of EGFR-TKIs, as well as the inappropriate TCM treatment principles to avoid when EGFR-TKIs are used in the treatment of NSCLC.

According to our finding, it seems that cooling-heat, rather than warming-yang TCM treatment principle, should 


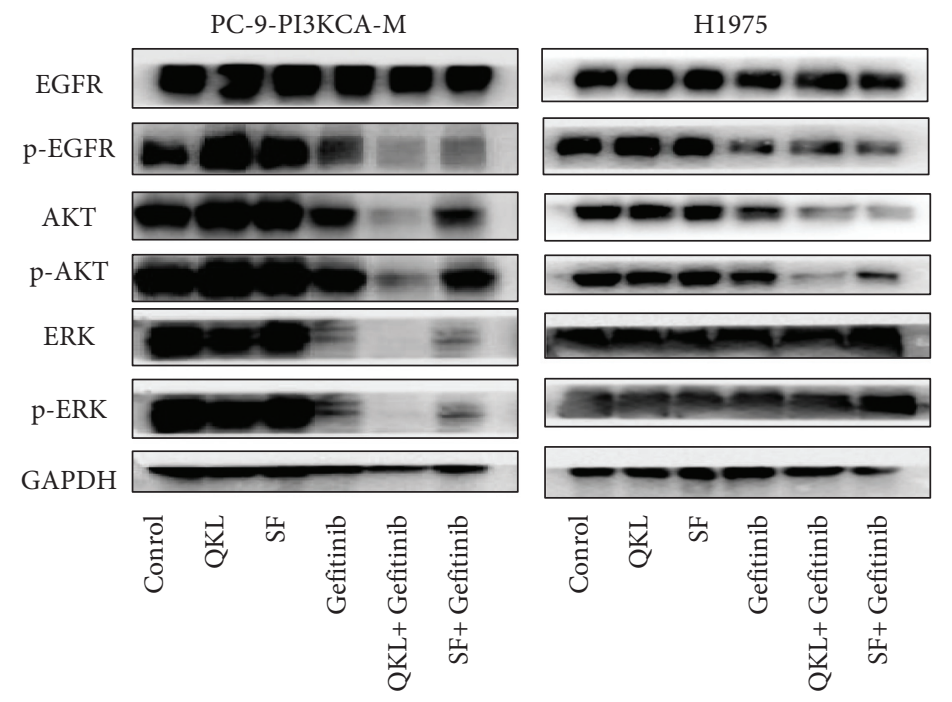

(a)
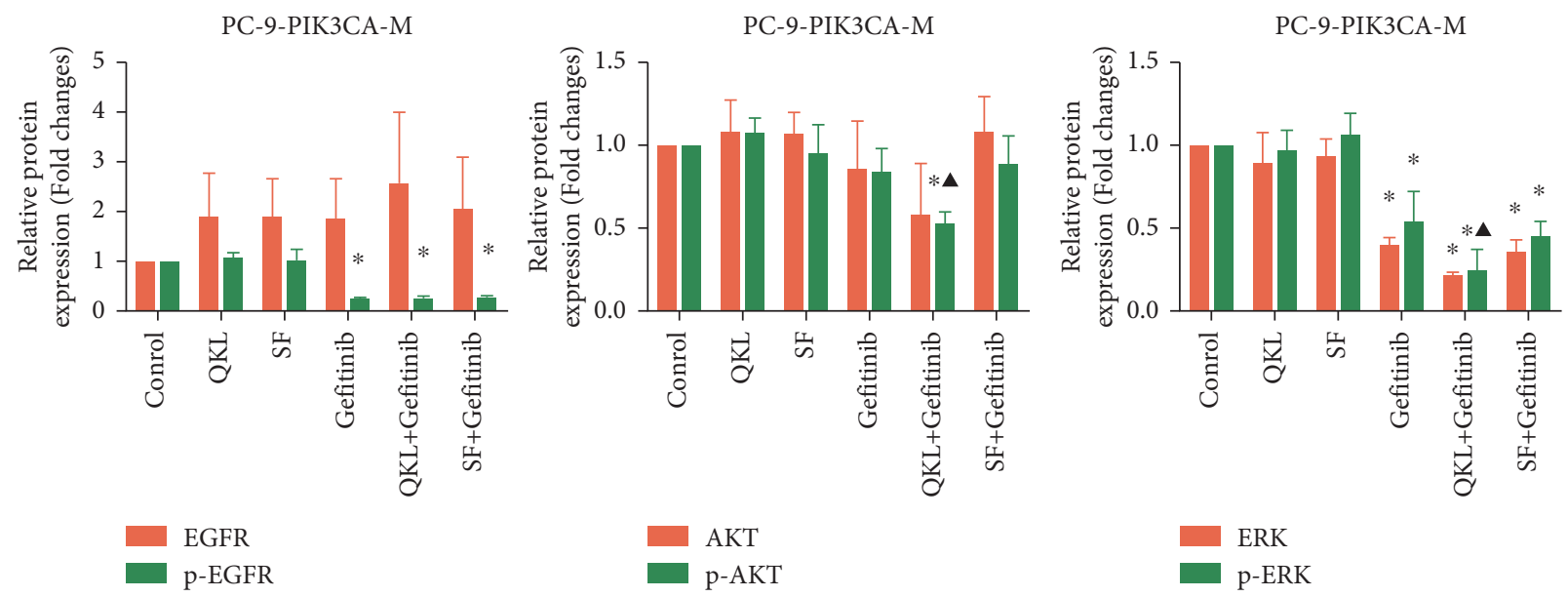

EGFR
p-EGFR

AKT
p-AKT

H1975
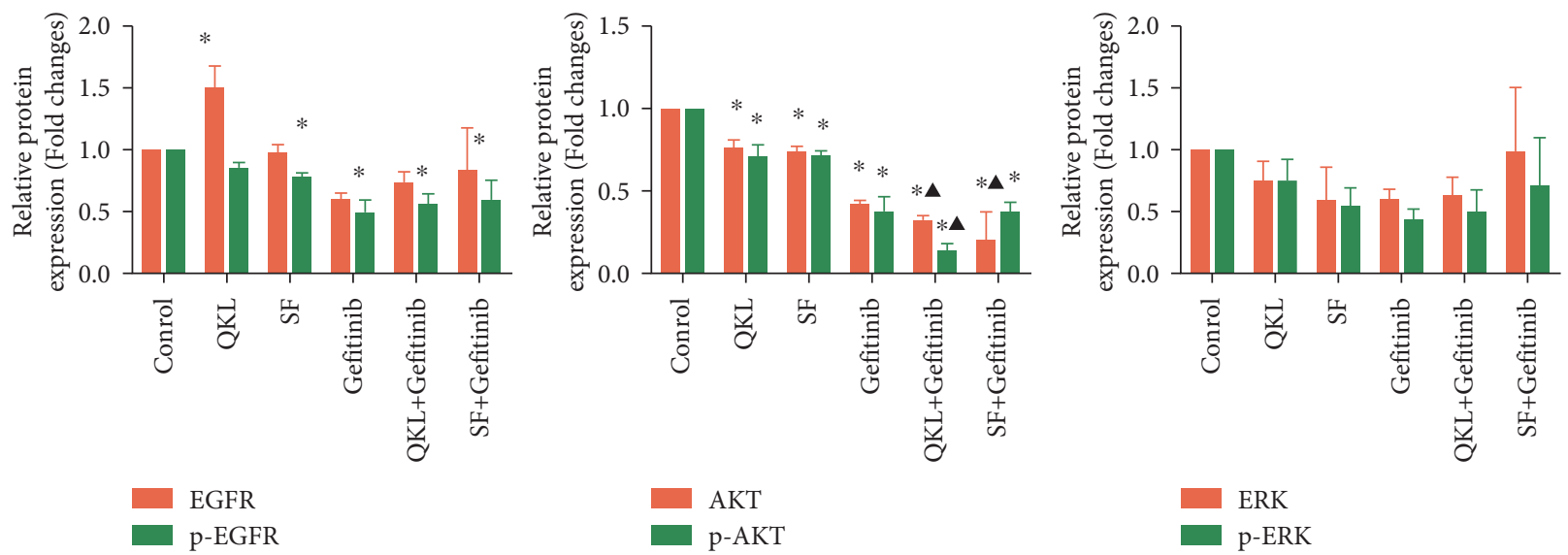

$\mathrm{AKT}$
$\mathrm{p}-\mathrm{AKT}$

ERK

(b)

FIgURE 4: Continued. 


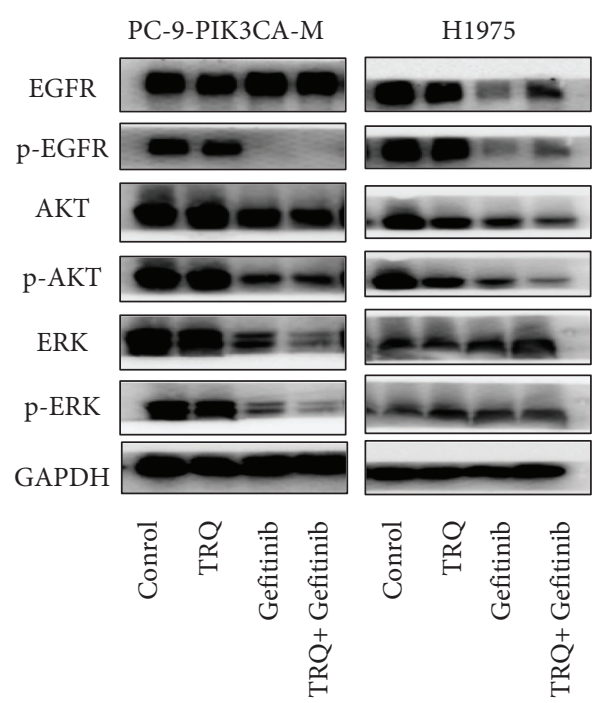

(c)
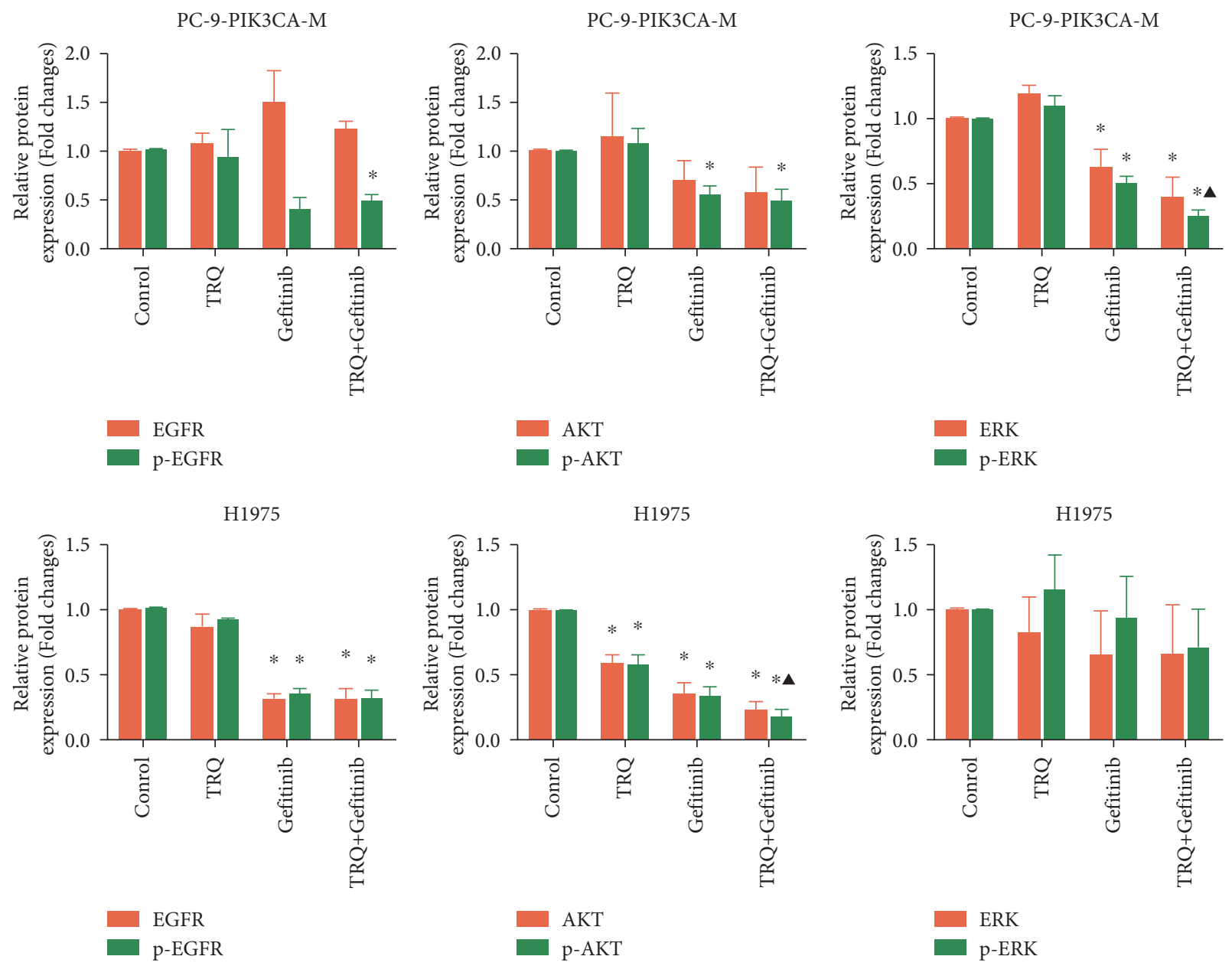

(d)

FIGURE 4: QKL/TRQ injection, not SF injection, enhanced the inhibition of the downstream signaling pathways of EGFR induced by gefitinib in vitro. (a) Western blot showing the expression of proteins related to the combination effect of gefitinib and QKL/SF in PC-9-PIK3CA-M and H1975 cell lines. (b) The effects of gefitinib and QKL/SF on EGFR, p-EGFR, AKT, p-AKT, ERK, and p-ERK protein expression in PC-9-PIK3CA-M cells and H1975 cells. (c) Western blot showing the expression of proteins related to the combination effect of gefitinib and TRQ in PC-9-PIK3CA-M and H1975 cell lines. (e) The effects of gefitinib and TRQ on EGFR, p-EGFR, AKT, p-AKT, ERK, and p-ERK protein expression in PC-9-PIK3CA and H1975 cells. Data are presented as the mean \pm standard deviation of three independent experiments. ${ }^{*} P<0.05$ vs. control. ${ }^{\wedge} P<0.05$ vs. gefitinib alone. QKL, Qing-kai-ling injection; SF, Shen-fu injection; TRQ, Tan-re-qing injection. 


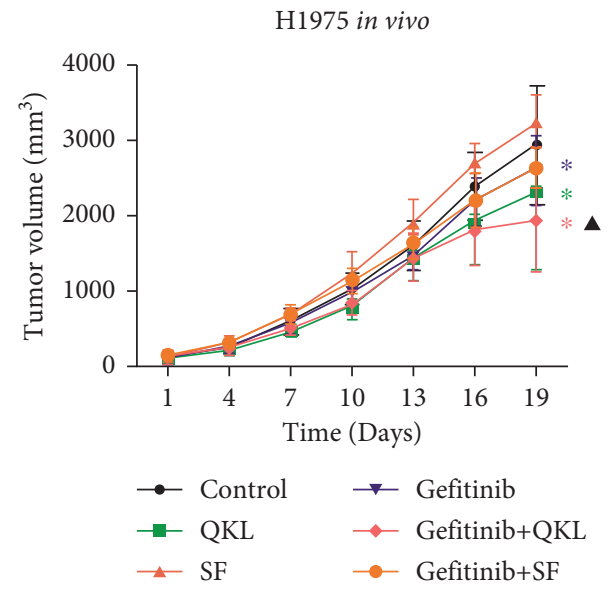

(a)

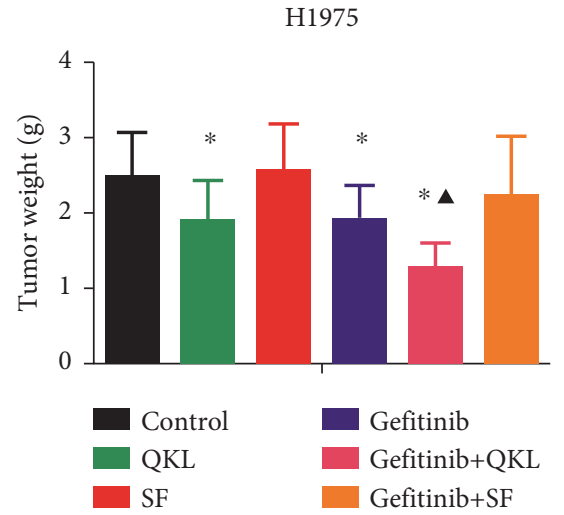

(b)
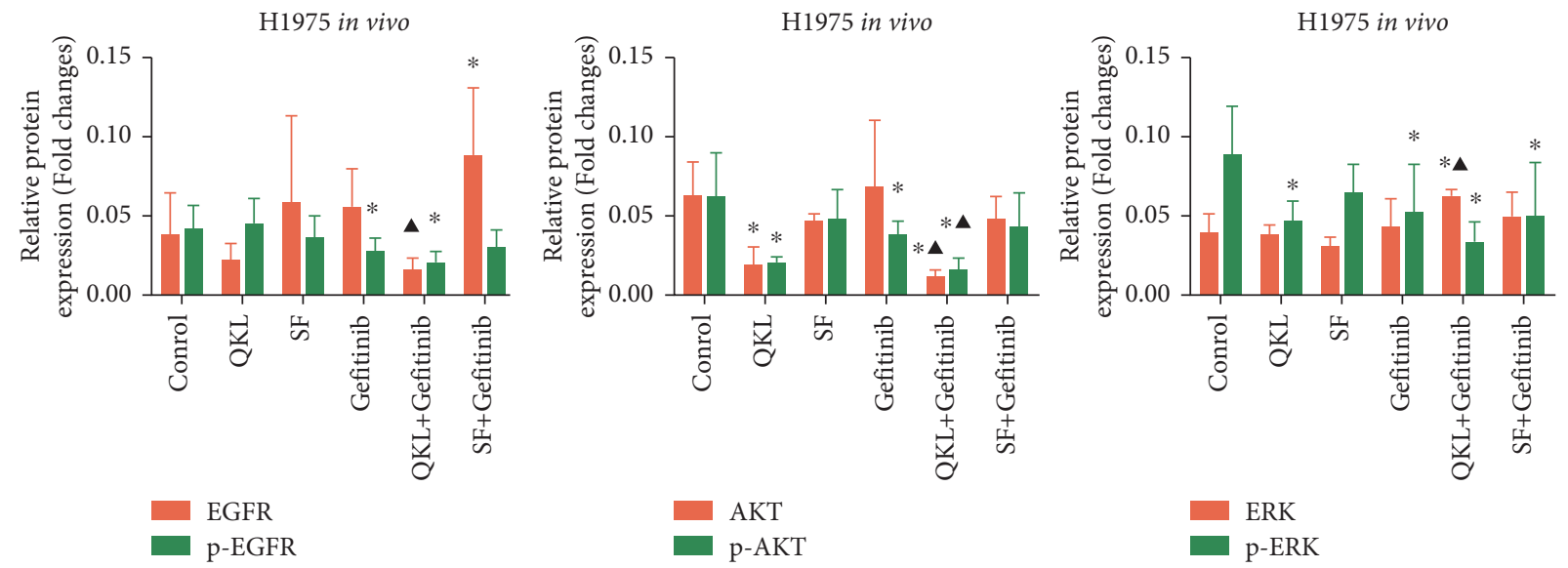

(c)

Figure 5: Continued. 


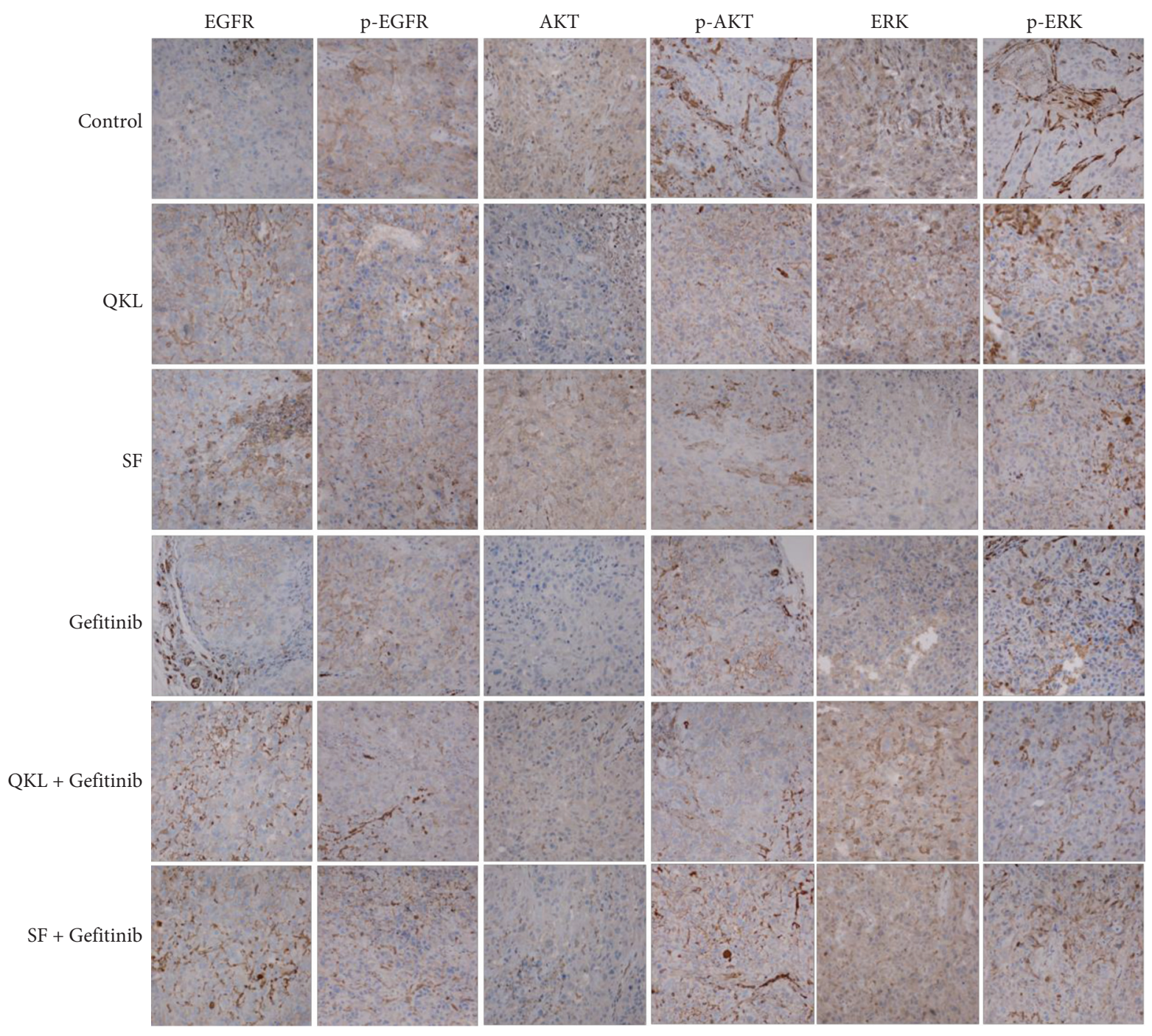

(d)

FIGURE 5: QKL oral solution, not SF decoction, and gefitinib synergistically inhibited the tumor growth via regulating p-AKT in vivo. (a) The tumor growth curve showing the anticancer effect of gefitinib and QKL/SF in H1975 xenograft transplanted nude mice. (b) The tumor weight showing the anticancer effect of gefitinib and QKL/SF in H1975 xenograft transplanted nude mice at day 19. (c) The statistics of EGFR, p-EGFR, AKT, p-AKT, ERK, and p-ERK protein expression in H1975 xenograft transplanted nude mice. (d) Immunohistochemical staining showing the effects of gefitinib and QKL/SF on the expression of EGFR, p-EGFR, AKT, p-AKT, ERK, and p-ERK protein $(\times 400$ magnifications). ${ }^{*} P<0.05$ vs. control group. ${ }^{\boldsymbol{\Lambda}} P<0.05$ vs. gefitinib group. QKL, Qing-kai-ling oral solution; SF, Shen-fu decoction.

be used when combined with EGFR-TKIs. Firstly, it was found that NSCLC patients with YC TCM syndrome were more likely to have the EGFR gene mutations than those with $Y H$ syndrome based on our previously published data [8]. Other team also found that heat syndrome was one of the most common syndromes in the NSCLC patients with EGFR gene mutation [22]. Since EGFR-TKIs are used in EGFR-mutated patients who are more common with YC syndrome, it might affect some warming-yang TCM syndrome. The most common side-effect of EGFR-TKIs, red acneiform rashes, also indicated its warming-yang effect. Because of the long-term need for warming-yang EGFR-TKIs taking, herbs with clear-heat function should be combined to get the balance between Yin and Yang and warm-yang herbs need to be avoided in case of the aggravation of $\mathrm{YH}$ syndrome type. Second, we also found that toxic-heat TCM syndrome was significantly associated with resistance to EGFR-TKI treatment (median PFS, 5.13 vs. 10.2 months). According to TCM theory, clearing-heat TCM herbs should be used in these toxic-heat patients, while warm-yang herbs should be avoided in this situation. Moreover, many TCM oncologists also use clearing-heat TCM decoctions to increase the effectiveness and reduce toxicity of EGFR-TKIs in clinical practice [23]. It was found that the classic clearing-heat TCM decoctions Yin-Qiao powder could significantly improve the skin rashes caused by EGFR-TKIs [24]. Compared with EGFR-TKIs alone, the clearing-heat TCM decoctions and EGFR-TKIs could significantly improve the patients' clinical curative effect [25]. The present study demonstrated that, in gefitinib resistant T790 M or PIK3CA comutated models, 
cooling-heat TCM prescription QKL and TRQ, but not warming-yang prescription SF solution, could significantly enhance the antitumor effects of gefitinib both in vitro and in vivo. Surprisingly, SF injection even antagonized the proapoptotic effect of gefitinib in vitro.

We also tried to find out the underlying mechanism of the synergic effect of QKL and TRQ to gefitinib. EGFR signaling is critical for several cellular functions including survival, proliferation, differentiation, and motility of cancer cells. EGFR activation transduces multiple signaling pathways, including the RAS/RAF/ERK pathway and the PI3K/ AKT/mTOR pathway [26]. Our findings demonstrated that AKT phosphorylation and ERK phosphorylation inhibition served an important role in the synergic effect of QKL/TRQ and gefitinib in PC-9-PIK3CA-M and H1975 cells in vitro and H1975 xenograft nude mice in vivo. Researchers also found that clearing-heat TCM decoctions or active ingredients from clearing-heat herbs can inhibit the growth of lung cancer via RAS/RAF/ERK pathway and the PI3K/AKT/ mTOR pathway. The cooling-heat medicine Compound Kushen injection significantly improved the sensitivity of gefitinib on less sensitive NSCLC cells in a combinatorial fashion through the PI3K/AKT/mTOR signaling pathway [27]. Solamargine, derived from cooling-heat medicine Solanum nigrum $L$, inhibited the growth of human lung cancer cells through inactivation of AKT signaling [28]. Ophiopogonin B, a saponin extracted from Ophiopogon japonicus (Thunb.) Ker Gawl., the cooling-heat herb, could suppress epithelial-mesenchymal transition through AKT phosphorylation [29]. Regulating the downstream signaling pathways of EGFR may act as important mechanisms underlying the cooling-heat medicine in exerting anticancer function in lung cancer.

There were several limitations in our present study. Firstly, we only used one or two drugs to present each of the treatment principles. Expansion of the observed synergic effect of QKL/TRQ, but not SF, with gefitinib to the effect of cooling-heat or warming-yang treatment principle should be cautious. We should further use other TCM patent prescriptions or decoction to present the cooling-heat or warming-yang treatment principle. Secondly, NSCLC cell lines with different EGFR-TKIs-resistant types should be applied to detect the combination effect of gefitinib and cooling-heat/warming-yang drugs. All these researches will be meaningful for guiding the principle of TCM therapies in combination with EGFR-TKIs or avoiding using them together with EGFR-TKIs to delay the resistance to EGFRTKIs or increase the sensitivity of EGFR-TKIs in EGFRTKIs-resistant NSCLC models.

\section{Conclusions}

In conclusion, QKL or TRQ, with cooling-heat TCM treatment principle, not warming-yang drug SF, increases the efficacy of gefitinib in the resistant NSCLC models. Furthermore, SF should be avoided to be used together with EGFR-TKIs. The influence of other drugs with cooling-heat or warming-yang TCM treatment principle warrants further study.

\author{
Abbreviations \\ EGFR-TKIs: Epidermal growth factor receptor tyrosine \\ kinase inhibitors \\ NSCLC: $\quad$ Non-small cell lung cancer \\ PC-9- PC-9-PIK3CA-mutation \\ PIK3CA-M: \\ QKL: $\quad$ Qing-kai-ling \\ SF: $\quad$ Shen-fu \\ TRQ: $\quad$ Tan-re-qing \\ HPLC: $\quad$ High performance liquid chromatography \\ UHPLC-MS: Ultra-high performance liquid \\ chromatography-mass spectrometry \\ technology \\ PFS: $\quad$ Progression-free survival \\ TCM: $\quad$ Traditional Chinese medicine \\ YC: $\quad$ Yin-cold \\ YH: Yang-heat \\ ORR: $\quad$ Objective response rate.
}

\section{Data Availability}

The data used in the study are available upon request by writing to the corresponding author.

\section{Disclosure}

The part of H1975 in vivo experiment of the manuscript was previously published as a preprint (https://www. researchsquare.com/article/rs-29063/v1).

\section{Conflicts of Interest}

The authors declare no conflicts of interest.

\section{Authors' Contributions}

Ya-ya Yu and Zhen-zhen Xiao performed the biological experiments in vitro. Ying Zou and Shuai Shi performed the biological experiments in vivo. Yi-hong Liu, Xue-song Chang, and Ya-dong Chen did the statistical analysis. Hai-bo Zhang conceived and supervised the study. Yan-juan Zhu and Ya-ya Yu drafted the manuscript. All the authors read and approved the final manuscript.

\section{Acknowledgments}

This work was supported by the National Natural Science Foundation of China (No. 81904004), the Guangzhou Municipal Science and Technology Bureau of China (No. 202102010307), the Foundation from Science and Technology Planning Project of Guangdong Province (Nos. 2017B030314166 and 2020B1212030006), and the Traditional Chinese Medicine Bureau of Guangdong Province (No. 20181128).

\section{References}

[1] C. Zhou, Y.-L. Wu, G. Chen et al., "Erlotinib versus chemotherapy as first-line treatment for patients with advanced 
EGFR mutation-positive non-small-cell lung cancer (OPTIMAL, CTONG-0802): a multicentre, open-label, randomised, phase 3 study," The Lancet Oncology, vol. 12, no. 8, pp. 735-742, 2011.

[2] M. Fukuoka, Y.-L. Wu, S. Thongprasert et al., "Biomarker analyses and final overall survival results from a phase III, randomized, open-label, first-line study of gefitinib versus carboplatin/paclitaxel in clinically selected patients with advanced non-small-cell lung cancer in Asia (IPASS)," Journal of Clinical Oncology, vol. 29, no. 21, pp. 2866-2874, 2011.

[3] M. Maemondo, A. Inoue, K. Kobayashi et al., "Gefitinib or chemotherapy for non-small-cell lung cancer with mutated EGFR," New England Journal of Medicine, vol. 362, no. 25, pp. 2380-2388, 2010.

[4] R. Rosell, E. Carcereny, R. Gervais et al., "Erlotinib versus standard chemotherapy as first-line treatment for European patients with advanced EGFR mutation-positive non-smallcell lung cancer (EURTAC): a multicentre, open-label, randomised phase 3 trial," The Lancet Oncology, vol. 13, no. 3, pp. 239-246, 2012.

[5] W. He and M. Cheng, "Meta-analysis on effectiveness and safety of traditional Chinese medicine combined with firstgeneration EGFR-TKI in treating advanced non-small cell lung cancer," Zhongguo Zhongyao Zazhi, vol. 42, no. 13, pp. 2591-2598, 2017.

[6] S.-W. Hwang, H.-S. Han, K. Y. Lim, and J.-Y. Han, "Drug interaction between complementary herbal medicines and gefitinib," Journal of Thoracic Oncology, vol. 3, no. 8, pp. 942-943, 2008.

[7] Z. Liu and W. Gao, "Leptomycin B reduces primary and acquired resistance of gefitinib in lung cancer cells," Toxicology and Applied Pharmacology, vol. 335, pp. 16-27, 2017.

[8] Y. J. Zhu, H. B. Zhang, L. R. Liu et al., "Yin-cold or yang-heat syndrome type of traditional Chinese medicine was associated with the epidermal growth factor receptor gene status in nonsmall cell lung cancer patients: confirmation of a TCM concept," Evidence-Based Complementary and Alternative Medicine, vol. 2017, Article ID 7063859, 7 pages, 2017.

[9] L. V. Sequist, J. C.-H. Yang, N. Yamamoto et al., "Phase III study of afatinib or cisplatin plus pemetrexed in patients with metastatic lung adenocarcinoma with EGFR mutations," Journal of Clinical Oncology, vol. 31, no. 27, pp. 3327-3334, 2013.

[10] X. Gao, L. Qin, Z. Zhang et al., "Deciphering biochemical basis of Qingkailing injection-induced anaphylaxis in a rat model by time-dependent metabolomic profiling based on metabolite polarity-oriented analysis," Journal of Ethnopharmacology, vol. 225, pp. 287-296, 2018.

[11] Q. Yin, B. Liu, C. Wu, J. Yang, C. Hang, and C. Li, "Effects of Shen-Fu injection on coagulation-fibrinolysis disorders in a porcine model of cardiac arrest," The American Journal of Emergency Medicine, vol. 34, no. 3, pp. 469-476, 2016.

[12] F. Zhang, L. Sun, J. Zhai et al., "The effect of tanreqing injection on the pharmacokinetics of sirolimus in rats," BioMed Research International, vol. 2019, Article ID 1854323, 7 pages, 2019.

[13] Y. J. Zhu, X. Qu, and D. D. Zhan, "Specific gene Co-variation acts better than number of concomitant altered genes in predicting EGFR-TKI efficacy in non-small-cell lung cancer," Clinical Lung Cancer, vol. 22, 2020.

[14] D. A. E. Cross, S. E. Ashton, S. Ghiorghiu et al., "AZD9291, an irreversible EGFR TKI, overcomes T790M-mediated resistance to EGFR inhibitors in lung cancer," Cancer Discovery, vol. 4, no. 9, pp. 1046-1061, 2014.
[15] Y. Gao, R. Qi, X. Zhang et al., "Qing-kai-ling injection induces immediate hypersensitivity reaction via the activation of anaphylatoxin C3," Frontiers in Pharmacology, vol. 10, p. 1524, 2019.

[16] C. Li, C. Zang, Q. Nie, B. Yang, B. Zhang, and S. Duan, "Simultaneous determination of seven flavonoids, two phenolic acids and two cholesterines in Tanreqing injection by UHPLC-MS/MS," Journal of Pharmaceutical and Biomedical Analysis, vol. 163, pp. 105-112, 2019.

[17] S. Reagan-Shaw, M. Nihal, and N. Ahmad, "Dose translation from animal to human studies revisited," The FASEB Journal, vol. 22, no. 3, pp. 659-661, 2008.

[18] Z. Liu, H. Li, Y. Fan et al., "Combination treatment with Rhizoma Paridis and Rhizoma Curcuma longa extracts and 10-hydroxycamptothecin enhances the antitumor effect in H22 tumor model by increasing the plasma concentration," Biomedicine \& Pharmacotherapy, vol. 83, pp. 627-634, 2016.

[19] W. Fang, Y. Huang, W. Gu et al., "PI3K-AKT-mTOR pathway alterations in advanced NSCLC patients after progression on EGFR-TKI and clinical response to EGFR-TKI plus everolimus combination therapy," Translational Lung Cancer Research, vol. 9, no. 4, pp. 1258-1267, 2020.

[20] M. K. Garcia, J. McQuade, R. Haddad et al., "Systematic review of acupuncture in cancer care: a synthesis of the evidence," Journal of Clinical Oncology, vol. 31, no. 7, pp. 952-960, 2013.

[21] Z.-L. Liu, W.-R. Zhu, W.-C. Zhou et al., "Traditional Chinese medicinal herbs combined with epidermal growth factor receptor tyrosine kinase inhibitor for advanced non-small cell lung cancer: a systematic review and meta-analysis," Journal of Integrative Medicine, vol. 12, no. 4, pp. 346-358, 2014.

[22] Q. L. Cui, Y. H. Hu, and D. Y. Ma, "Non-small cell lung cancer patients constitution of traditional Chinese medicine syndrome and the correlation of EGFR gene research," Lishizhen Medicine and Materia Medica Research, vol. 28, no. 6, pp. 1516-1518, 2017.

[23] S.-K. Liang, J.-C. Ko, J. C.-H. Yang, and J.-Y. Shih, "Afatinib is effective in the treatment of lung adenocarcinoma with uncommon EGFR p.L747P and p.L747S mutations," Lung Cancer, vol. 133, pp. 103-109, 2019.

[24] W. G. Shi, Y. M. Zhou, and L. S. He, "Clinical-Observation of YinqiaoSan in treating rash induced by iressa," China Journal of Chinese Medicine, vol. 29, no. 194, pp. 954-955, 2014.

[25] S. Chen, Z. Zhang, and X. Zhang, "Effect of yiqi tongluo jiedu decoction combined with EGFR- TKI targeted therapy on patients with non-small cell lung cancer and its effect on patients' quality of life," Jilin Medicine, vol. 38, no. 12, pp. 2288-2290, 2017.

[26] S. Sigismund, D. Avanzato, and L. Lanzetti, "Emerging functions of the EGFR in cancer," Molecular Oncology, vol. 12, no. 1, pp. 3-20, 2018.

[27] J. Zhang, Z. Qu, H. Yao et al., "An effective drug sensitizing agent increases gefitinib treatment by down regulating PI3K/ Akt/mTOR pathway and up regulating autophagy in nonsmall cell lung cancer," Biomedicine \& Pharmacotherapy, vol. 118, Article ID 109169, 2019.

[28] Y. Chen, Q. Tang, J. Wu, F. Zheng, L. Yang, and S. S. Hann, "Inactivation of PI3-K/Akt and reduction of SP1 and p65 expression increase the effect of solamargine on suppressing EP4 expression in human lung cancer cells," Journal of Experimental \& Clinical Cancer Research, vol. 34, no. 1, p. 154, 2015.

[29] C. Hu, R. Jiang, Z. Cheng et al., "Ophiopogonin-B suppresses epithelial-mesenchymal transition in human lung adenocarcinoma cells via the linc00668/miR-432-5p/EMT Axis," Journal of Cancer, vol. 10, no. 13, pp. 2849-2856, 2019. 